\title{
An unusual localized laterally spreading tumor
}

A 54-year-old man was referred for a screening colonoscopy. He had no family history of colorectal cancer and no significant prior history, except for mild arterial blood hypertension that was well controlled with beta blockers (atenolol $50 \mathrm{mg} /$ day). Physical examination results were normal and colon preparation was uneventful. Colonoscopy showed several diverticula in the left colon; otherwise findings were normal up to the terminal ileum. During scope withdrawal, careful examination of the sigmoid colon revealed a flat-type laterally spreading tumor with a small area of protruding lesion ( $\bullet$ Fig. 1 ). A better look at the lesion with colonic distension showed that the tumor involved the entire surface of a single diverticulum ( $\bullet$ Figs. 2,3). Because of the location and the low risk of the surgery to the patient, the lesion was tattooed ( $\bullet$ Fig. 4 gray areas) and the patient was referred for a laparoscopic left colectomy, which was successfully performed with no complications. Histological analysis revealed the presence of an adenoma with low grade dysplasia. The patient is now having regular colonoscopic surveillance.

Endoscopy_UCTN_Code_CCL_1AD_2AB

Competing interests: None

Erika P. Macedo, Fernanda P. Martins, Gustavo A. de Paulo, Angelo P. Ferrari

Hospital Israelita Albert Einstein,

Endoscopy Unit, São Paulo, Brazil

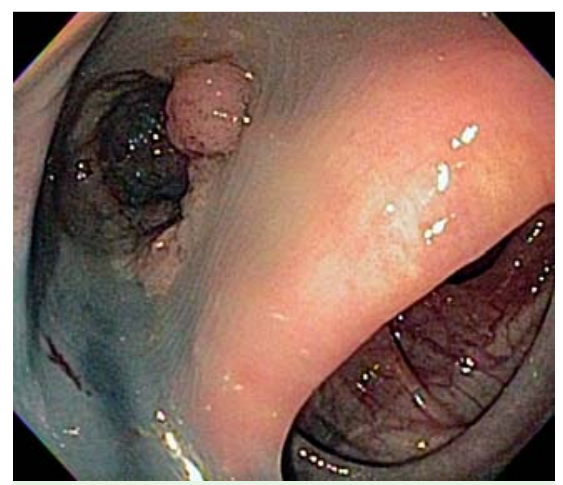

Fig. 1 Lesion from a laterally spreading tumor in the sigmoid colon of a 54-year-old man, observed during screening. First view of the lesion, showing a granular portion outside of the diverticulum.

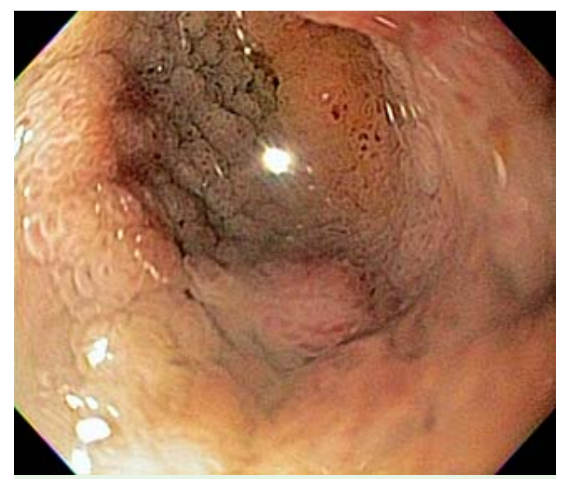

Fig. 3 Vascular pattern in a laterally spreading tumor as observed using narrow band imaging.

Bibliography

Dol http://dx.doi.org/

10.1055/s-0034-1391778

Endoscopy 2015; 47: E212

(c) Georg Thieme Verlag KG

Stuttgart · New York

ISSN 0013-726X

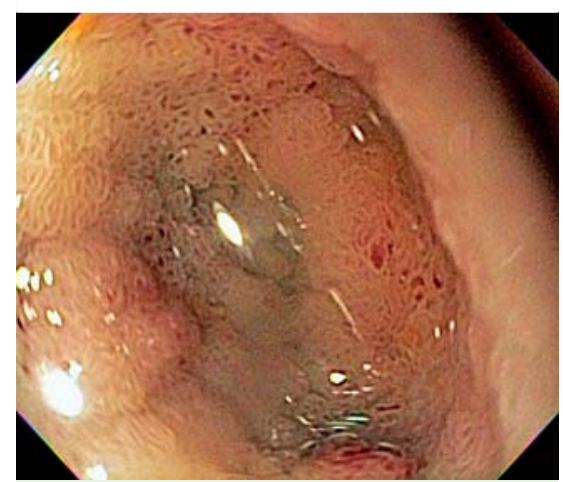

Fig. 2 A closer view showing the laterally spreading tumor inside the diverticulum.

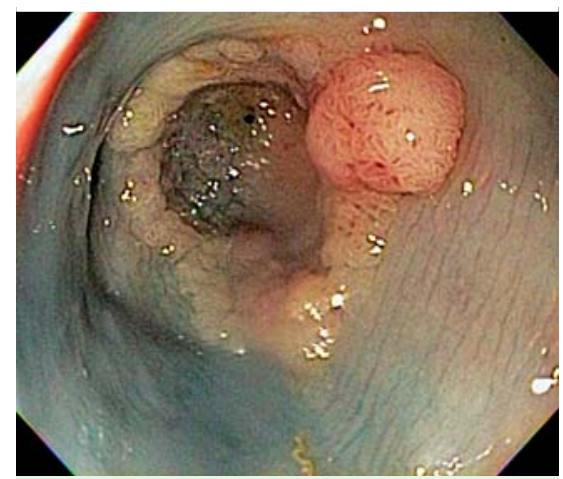

Fig. 4 Final view of the laterally spreading tumor, showing the endoscopic tattoo.

\section{Corresponding author}

Angelo Ferrari, MD

Endoscopy Hospital Israelita Albert Einstein

Rua Dr. Bacelar, 317 ap 231

São Paulo 04026-001

Brazil

Fax: +55-11-55716454

angelo.ferrari@outlook.com 